\section{Die Erfassung des Cravings bei Alkoholabhängigen}

Zusammenfassung: Craving, also das starke, unbezwingbare Verlangen nach einer psychotropen Substanz, wird als ein zentrales Merkmal der Sucht betrachtet. Im Folgenden werden verschiedene Methoden und (in deutscher Version) vorliegende Instrumente zur Erfassung des Cravings bei Alkoholabhängigen beschrieben sowie Ergebnisse zum Alkoholverlangen aus einer Studie an 103 alkoholabhängigen Patienten während einer stationären Entwöhnungsbehandlung dargestellt.

Schlüsselwörter: Craving - Obcessive-Compulsive Drinking Scale - Craving Beliefs Questionnaire - Inventory of Drug Taking Situations - Lübecker Craving Skala - Alkoholreagibilität

The Assessment of Craving in Alcohol Dependent Persons: - Craving - the strong desire to drink alcohol - is considerered to be a central phenomenon related to substance dependence. The most relevant rating scales for craving (German versions) are described. There are results from a craving study of 103 alcohol dependent patients during their inpatient treatment.

Key words: Craving - Obcessive-Compulsive Drinking Scale - Craving Beliefs Questionnaire - Inventory of Drug Taking Situations - Luebeck Craving Scale - Alcohol Reagibility

\section{Einführung}

Der Begriff des Cravings ist bislang nur unscharf definiert. Craving ist ein Konstrukt, mit dem man sich von wissenschaftlicher Seite beschäftigt, welches aber auch im Erleben vieler betroffener Suchtmittelabhängiger (im Sinne von „Jieper“, „Janker“ oder „Saufdruck“) von zentraler Bedeutung ist. Watzl und Gutbrod [1] weisen zu Recht darauf hin, dass mit dem „Verlangen nach Alkohol“ sehr unterschiedliche Prozesse gemeint sind. Das Craving kann beim Entzug im Zusammenhang mit dem Wunsch der Änderung von als unangenehm erlebten Wirkungen des Suchtmittels auftreten, wobei gleichzeitig Gewissheit besteht, dass der erneute Suchtmittelkonsum die negativen Folgen auch tatsächlich

Suchttherapie 2001; 2: 14-24

(c) Georg Thieme Verlag Stuttgart · New York ISSN 1439-9903
Clemens Veltrup ${ }^{1}$, Franziska Einsle ${ }^{2}$,

Johannes Lindenmeyer ${ }^{3}$, Tilman Wetterling ${ }^{4}$, Klaus Junghanns ${ }^{5}$

1 Therapieverbund Ostsee

2 Technische Universität Dresden

${ }^{3}$ salus Klinik Lindow

${ }^{4}$ Klinik für Psychiatrie und Psychotherapie der J.-W.-Goethe Universität Frankfurt

${ }^{5}$ Klinik für Psychiatrie und Psychotherapie im Universitätsklinikum Lübeck

beseitigt. In diesem Fall wird von einem physiologischen Craving (sekundären Craving) gesprochen.

Das Craving kann als subjektives Verlangen, vor allem aber während längerer konsumfreier (abstinenter) Phasen, auftreten; man spricht in diesem Zusammenhang auch vom „primären“, „psychischen“ bzw. „symbolischen“ Craving. Craving tritt nach dem ersten Schluck Alkohol nach einer Phase der Konsumfreiheit auf und wird damit zu einem wichtigen Faktor bei der Fortsetzung des Substanzkonsums im Zusammenhang mit einem „Rückfall“.

Craving ist in hohem Maß zustandsabhängig. Es kann zeitweise gar nicht oder kaum, dann wieder subjektiv als ausgesprochen stark erlebt werden [2].

Tiffany [3] beschreibt Craving als einen kognitiven Prozess der Annäherung und Orientierung an psychotrope Substanzen. Marlatt [4] betont auch die affektiv-motivationale Komponente des Cravings. Beck, Wright, Newman und Liese [5] definieren das Verlangen nach einer psychotropen Substanz als starken Wunsch, das Suchtmittel zu konsumieren und die Wirkung der Substanz zu erleben. Von Anton [6] wird der Zwangscharakter des Verlangens betont: Die psychotrope Substanz wird vermisst, es bestehen eine starke gedankliche Einengung auf die psychotrope Substanz, eine beständige Wahrnehmung des Verzichts auf die Substanz und erhebliche Schwierigkeiten, dem Verlangen zu widerstehen.

Diese Aspekte des Cravings finden sich auch in der Operationalisierung des Phänomens in der ICD-10 [7], die Craving als starken Wunsch oder eine Art Zwang beschreibt, eine psychotrope Substanz zu konsumieren.

Das Craving ist ähnlich wie das Schmerzempfinden nur subjektiv erlebbar und kann zudem als Auslöser für automatisiertes Verhalten angesehen werden. Dabei spielen, wie beschrieben, Prozesse von kognitiver und affektiver Einengung eine große Rolle.

Es bestehen unterschiedliche Erklärungsansätze, wie Craving entsteht. Die meisten der vorliegenden Modelle beziehen sich auf das Verlangen nach Alkohol. Moderne biochemische Erklärungsansätze [8] versuchen, das Auftreten von Craving nach längerer Abstinenz zu erklären. Dabei steht das Belohnungssystem im ZNS im Mittelpunkt der Überlegungen. Bei verminderter Aktivität dieses Systems kann es zu Frustrationen oder auch zum Craving kommen [9]. Wetterling, Veltrup und Junghanns [10] betonen, dass Alkohol akute und 
Der folgende Fragebogen beschäftigt sich mit Gedanken, Vorstellungen, Antrieben oder Bildern, die etwas mit dem Trinken von Alkohol zu tun haben. Gehen Sie die Fragen einzeln durch und entscheiden Sie sich jeweils für eine Antwortalternative!

1. Wenn Sie keinen Alkohol trinken, wie viel Zeit wird dann von Vorstellungen oder Gedanken, die etwas mit dem Trinken zu tun haben, beansprucht?

0 keine

1 weniger als eine Stunde am Tag

2 1-3 Stunden am Tag

3 4-8 Stunden am Tag

4 mehr als 8 Stunden am Tag

2. Wie häufig treten solche Gedanken oder Vorstellungen auf?

0 niemals

1 nicht öfter als achtmal am Tag

2 öfter als achtmal am Tag, aber der größte Teil des Tages ist frei davon

3 mehr als achtmal am Tag und während der meisten Stunden am Tag

4 die Gedanken treten so häufig auf, dass man sie nicht mehr zählen kann. Es vergeht kaum eine Stunde am Tag, in der sie nicht auftreten.

3. Wie stark wird Ihre Arbeit oder Ihr soziales Verhalten von diesen Vorstellungen oder Gedanken beeinflusst? Gibt es etwas, was Sie wegen dieser Vorstellungen oder Gedanken nicht mehr tun können?

(Falls Sie gerade keiner beruflichen Arbeit nachgehen: Wie sehr würde das Auftreten dieser Gedanken Ihre Arbeit oder Ihr soziales Verhalten beeinflussen?)

.. 00 Die Gedanken an Alkohol beeinflussen mich überhaupt nicht, ich bin völlig normal.

.. 1 Die Gedanken an Alkohol beeinflussen mein soziales und berufliches

Verhalten in geringem Maße, mein Auftreten insgesamt ist jedoch nicht beeinträchtigt.

2 Die Gedanken an Alkohol beeinflussen mein soziales und berufliches

Verhalten, aber ich komme damit zurecht.

3 Die Gedanken an Alkohol beeinflussen mein soziales und berufliches

Verhalten ganz wesentlich.

$4 \quad$ Die Gedanken an Alkohol verhindern bei mir ein angemessenes soziales und berufliches Verhalten völlig.

4. Wie stark ist der Leidensdruck, der bei Ihnen durch diese Vorstellungen bzw. Gedanken hervorgerufen wird, wenn Sie keinen Alkohol trinken?

0 nicht vorhanden

1 gering, selten und nicht besonders störend

2 mittelmäßig, häufig und störend, ich kann aber damit zurechtkommen

3 stark, sehr häufig und sehr störend

4 äußerst stark, fast dauernd, sie erzwingen Untätigkeit

5. Wie viel Anstrengung unternehmen Sie, um diesen Gedanken widerstehen zu können, wenn Sie keinen Alkohol trinken? (Bewerten Sie das Ausmaß Ihrer Bemühungen um Widerstand gegen jene Gedanken, nicht den Erfolg oder Misserfolg, den Sie dabei haben!)

. $\quad 0 \quad$ Ich habe so selten derartige Vorstellungen, dass es nicht notwendig ist, dagegen anzugehen. Wenn sie aber auftauchen, bemühe ich mich, sie völlig zu unterdrücken.

1 Meistens versuche ich, jene Gedanken zu unterdrücken.

2 Ich unternehme einige Anstrengungen, um jene Gedanken zu unterdrücken

3 Ich gebe derartigen Vorstellungen nach, ohne etwas dagegen zu tun, aber ich habe dabei etwas Widerwillen.

4 Ich gebe allen solchen Vorstellungen bereitwillig nach.

6. Wie viel Erfolg haben Sie, wenn Sie jene Gedanken dann unterdrücken oder zerstreuen?

0 Ich unterdrücke solche Gedanken stets vollständig.

1 Mit einiger Anstrengung und Konzentration kann ich gewöhnlich jene Gedanken unterdrücken.

2 Manchmal kann ich jene Gedanken unterdrücken.

3 Ich kann jene Gedanken nur selten unterdrücken und kann meine Aufmerksamkeit nur mit Mühe davon ablenken.

4 Ich kann meine Aufmerksamkeit nur selten für einen Augenblick von jenen Gedanken ablenken.

7. Wie viele „Drinks“ nehmen Sie täglich zu sich?

$0 \quad$ keinen

1 weniger als einen am Tag

2 1-2 Drinks am Tag

Abb. 1 Obsessive Compulsive Drinking Scale (OCDS). 
Tab. 1 Inventory of Drug Taking Situations (Faktor: plötzliches Verlangen)

\begin{tabular}{|c|c|c|c|}
\hline Im letzten Jahr trank ich Alkohol, ... & Item-Nr. & $\begin{array}{l}\text { Schwierig- } \\
\text { keitsindex }\end{array}$ & $\begin{array}{l}\text { Trennschärfe- } \\
\text { koeffizient }\end{array}$ \\
\hline wenn ich an einem Ort war, an dem ich früher Alkohol gekauft oder getrunken hatte. & 06 & 0,43 & 0,5587 \\
\hline $\begin{array}{l}\text { wenn ich unerwartet eine Flasche meines Lieblingsgetränks fand oder zufällig etwas sah, } \\
\text { das mich an Alkohol erinnerte. }\end{array}$ & 16 & 0,42 & 0,5831 \\
\hline $\begin{array}{l}\text { wenn ich beim Rauchen einer Zigarette oder der Einnahme von bestimmten Medikamenten } \\
\text { an das Trinken von Alkohol dachte. }\end{array}$ & 26 & 0,30 & 0,5910 \\
\hline wenn ich jemanden über seine Trinkzeiten reden hörte. & 36 & 0,19 & 0,6278 \\
\hline $\begin{array}{l}\text { wenn ich mich daran erinnerte, wie gut es täte, etwas Alkohol zu trinken oder beschwipst } \\
\text { zu werden. }\end{array}$ & 46 & 0,46 & 0,5517 \\
\hline
\end{tabular}

chronisch pharmakologische Prozesse im ZNS auslösen kann. Es werden u.a. folgende zwei Modelle diskutiert: 1. Es besteht eine Unterstimulation des Belohnungssystems. So beschreiben Wetterling, Veltrup und Junghans [11], dass bestimmte Gruppen von Alkoholabhängigen einen erniedrigten $\beta$-Endorphin-Spiegel aufweisen. Dieser niedrige Spiegel kann eine der Ursachen für den Missbrauch der Substanz bei dieser Subgruppe sein, da $\beta$-Endorphin eine wichtige regulatorische Funktion innerhalb des Belohnungssystems einnimmt und durch Alkohol stimuliert werden kann. Des Weiteren fand man ein Serotonindefizit bei abstinenten Alkoholabhängigen, welches mit verschiedenen psychischen Störungen in Verbindung gebracht wird. Weiter fanden Wetterling, Veltrup und Junghanns [11], dass starkes Alkoholcraving bei niedrigen Liquorwerten des Hauptabbauprodukts von Noradrenalin (MOPEG) auftritt. 2. In diesem Modell wird die verringerte Sensitivität bestimmter Neuronenverbände herausgestellt, welche durch den Konsum von Alkohol geschädigt wurden. Wetterling, Veltrup und Junghanns [8] konnten zeigen, dass Kondensationsprodukte aus Alkoholabbauprodukten, welche in früher Abstinenz verstärkt gebildet worden sind, langfristig
Neuronen schädigen, was eine verminderte Erregbarkeit mit sich bringt.

Es gibt auch lerntheoretische Erklärungsansätze. Beck et al. [5] betonen das Erleben der positiven Wirkung des Alkohols als wichtigen Faktor für die Entstehung von Verlangen. Je häufiger positive Wirkungen erlebt worden sind, umso stärker entwickelt sich beim Fehlen des Alkohols ein starkes Verlangen nach Einnahme der Substanz. Beim Verspüren des Wunsches entsteht ein innerer Druck; das Erleben von Verlangen ist mit einem starken Aufforderungscharakter verbunden, dem nur schwer zu widerstehen ist. Vor dem Hintergrund allgemeiner Grundüberzeugungen entwickeln sich suchtspezifische Grundannahmen (z.B. „Alkohol macht mich lockerer"), die ein Verlangen erzeugen, welche wiederum erlaubniserteilende Gedanken in Gang setzen (z.B. „Dieses eine Mal wird mir doch nicht schaden“) sowie entsprechende Verhaltensweisen, die den Konsum des Suchtmittels wahrscheinlicher machen. Verlangen kann auch durch bestimmte Erinnerungen hervorgerufen werden, die wiederum bestimmte Grundannahmen aktivieren. Weiterhin

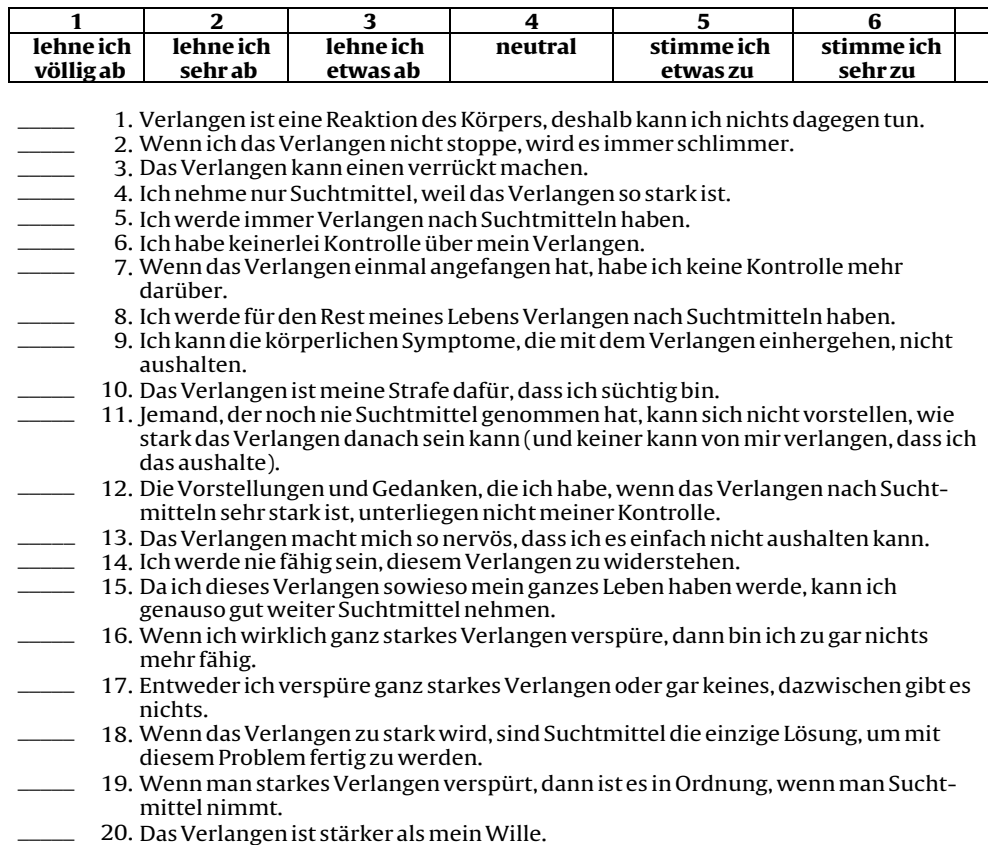

Abb. 2 Craving Beliefs Questionnaire (CBQ). 
betonen Beck et al. [5], dass es auch Grundüberzeugungen zum Verlangen gibt, die im Sinne eines Attributionsstils wirken ( z. B. „Ich werde niemals dem Verlangen widerstehen können“) und so möglicherweise das aktuelle Verlangen nach einer psychotropen Substanz in einer neutralen Situation beeinflussen. Eine andere Hypothese stellt die Erfahrung negativer Folgen bei der Abstinenz in den Mittelpunkt. So wird nach länger andauerndem Konsum der Belohnungseffekt immer kleiner, der Bestrafungseffekt bei Konsumbeendigung aber immer größer. Verlangen entsteht nun aus dem Bedürfnis, sich besser zu fühlen.

Ludwig, Wikler und Stark [12] postulieren, dass Verlangen ein kognitives Korrelat einer konditionierten Reaktion ist. Tiffany [3] geht davon aus, dass Craving als Resultat eines Wechselspiels von automatisierten und kontrollierten Handlungsschemata entsteht. Handlungsschemata sind schnell und effizient in spezifischen Situationen anwendbar. Craving ist eine Reaktion auf eine Blockade der Handlungsschemata: Eine Person befindet sich in einer typischen Trinksituation, hat aber eine Abstinenzentscheidung getroffen. Bei dem Bemühen, diese Entscheidung aufrechtzuerhalten, entstehen Reaktionsmuster, die sich aus den Komponenten des früheren Konsumverhaltens und alternativen Handlungsschemata zusammensetzen. Nur durch bestimmte nicht automatische Bewältigungsprozesse kann diese Situation überwunden werden.

\section{Verfahren zur Erfassung des Cravings}

Es lassen sich folgende Messmethoden zur Bestimmung des Cravings unterscheiden [13]:

1. subjektive Indizes (Selbstbeschreibungs- und -beurteilungsskalen),

2. Verhaltensbeobachtungen (z. B. Geschwindigkeit des Konsums, Konsummenge etc.)

3. physiologische Hinweise (z. B. Handtremor, Hautleitfähigkeit, Herzrate, Speichelfluss etc.)

An dieser Stelle sollen v.a. die Selbstbeschreibungsskalen dargestellt werden.

Sehr häufig werden visuelle Analogskalen oder skalierte Skalen zur Erfassung des Cravings verwendet. So erhält der Patient beispielsweise $10 \mathrm{~cm}$ lange Skalen mit den Polen „kein Verlangen nach Alkohol“ und „unwiderstehlicher Drang nach Alkohol“.

Die meisten dieser Skalen besitzen unklare Reliabilitäten und Validitäten [14]. Ein mittlerweile sehr verbreitetes Instrument zur Erfassung des Cravings ist die „Obsessive Compulsive Drinking Scale“ von Anton, Moak und Laham [6,15]. Dieses Instrument ist in Abb. 1 dargestellt.

Der Fragebogen von Beck et al. [5] überprüft Grundüberzeugungen zum Craving (Craving Beliefs Questionnaire), die ihrerseits Einfluss auf das Umgehen mit dem Verlangen haben. In Abb. 2 ist das aus 20 Items bestehende Instrument dargestellt.

Auch im aus 50 Items bestehenden „Inventory of Drug Taking Situations (IDTSA)“ von Annis, Martin und Graham [16], welches in einer geprüften deutschen Version von Linden- meyer und Florin [17] vorliegt, wird das „plötzliche Verlangen“ als einer von acht Risikofaktoren für den Konsum von psychotropen Substanzen erfasst und als intrapersonale Versuchungssituation kategorisiert. In Tab. 1 sind die entsprechenden Items und Kennwerte der Skala aus der Untersuchung von Lindenmeyer und Florin dargestellt. Die interne Konsistenz dieser Skala betrug bei Lindenmeyer und Florin $0,77[17]$.

Die Lübecker Craving-Skala (LCS) misst folgende Aspekte des Alkoholverlangens in einem definierten Zeitraum von 30 Tagen vor der Selbstbeurteilung: 1. Stärke des Verlangens ( 0 = starke Abneigung gegen Alkohol, $10=$ wahnsinnige Lust auf Alkohol), 2. Häufigkeit des Auftretens von Verlangen ( 10 = fast durchgängig vom Aufstehen bis zum Einschlafen, $1=$ nie), 3. Zeitpunkt des Auftretens von Verlangen (morgens, mittags, nachmittags etc.), 4. Häufigkeit des Auftretens von Verlangen an bestimmten Orten und Plätzen, 5. Non-Craving Situationen (freie Frage).

In einem weiteren Abschnitt werden schwerpunktmäßig die affektiven Aspekte des Cravings behandelt. Es wird erfragt, unter welchen Bedingungen Alkoholverlangen gespürt worden ist und ob dann auch üblicherweise Alkohol getrunken worden ist. In Abb. 3 sind die 33 Aussagen zu Auftretensbedingungen des Cravings dargestellt.

Dieses Selbstbeurteilungsinstrument ist in einer Studie von 146 entzugsbehandelten Alkoholabhängigen [18] erprobt worden. Es konnten vier Subskalen (Faktoren) identifiziert werden (vgl. Tab. 2).

In einer empirischen Untersuchung von Veltrup und Wetterling [19] konnte bei 161 alkoholabhängigen Patienten, die im Rahmen einer qualifizierten stationären Entzugsbehandlung an einer dreiwöchigen gruppentherapeutischen Motivationsbehandlung in der psychiatrischen Klinik der Medizinischen Universität zu Lübeck (Direktor: Prof. Dr. H. Dilling) teilnahmen, ein Zusammenhang mit dem Schweregrad des Auftretens von Craving in den letzten 30 Tagen vor der stationären Aufnahme und dem Trinkverhalten vier Monate nach Abschluss der Behandlung festgestellt werden (vgl. Tab.3). Die nach Abschluss der Therapie durchgängig Abstinenten erlebten vor der Behandlung ein weniger ausgeprägtes Craving, welches sich nach vier Monaten Abstinenz noch deutlich reduzierte, während bei den nachklinischen Rückfällen ein vor der Behandlung ausgeprägteres Alkoholverlangen festzustellen war, welches nachklinisch auch geringer wurde.

Zur Erfassung von physiologischen Parametern eignet sich der Alkoholreagibilitätstest, der auch in Deutschland angewendet wird. Die folgende Beschreibung stellt das Vorgehen in der Salus Klinik Lindow (Direktor: Dr. J. Lindenmeyer) dar. Der Alkoholreagibilitätstest besteht aus mehreren Phasen: 1. Übungsphase, 2. Konfrontation mit Wasser, 3. Konfrontation mit Alkohol. Die Untersuchung wird als Einzeltest durchgeführt. Die Dauer beträgt ca. 45 Minuten. Der Versuchsleiter befindet sich während der gesamten Untersuchungszeit mit dem Probanden im Untersuchungslabor. Die Instruktionen werden dem Probanden teils mündlich vom Versuchsleiter, teils vom Tonband abgespielt. In Abb. 4 ist das Vorgehen kurz skizziert. Neben der Messung der Gewichtsdifferenz wird 
Tab. 2 Craving-Subskalen der Lübecker Craving-Skala

\begin{tabular}{lll}
\hline Subskala & $\begin{array}{l}\text { Anzahl } \\
\text { der Items }\end{array}$ & Beispiel-Items \\
\hline gedrückte Stimmungslage & 10 & "Ich hatte Verlangen nach Alkohol, wenn ich mich in einer depressiven Stimmung fühlte.“ \\
gehobene Stimmungslage & 05 & "Ich hatte Verlangen nach Alkohol, wenn ich in guter Stimmung war." \\
$\begin{array}{ll}\text { Ärger und Anspannung } \\
\text { Zufriedenheit und Entspannung }\end{array}$ & 05 & "Ich hatte Verlangen nach Alkohol, wenn ich mich geärgert hatte oder wütend war.“ \\
& 03 & "Ich hatte Verlangen nach Alkohol, wenn ich zufrieden und entspannt war."
\end{tabular}

auch geprüft, inwieweit Probanden körperliche Veränderungen bei der Konfrontation mit Wasser bzw. Alkohol erleben (z.B. Zittern, Schwitzen, Schwindelgefühl, Übelkeit) sowie kognitive Reaktionen (z.B. Erinnerung an Trinkzeiten, sich wie betrunken fühlen).

\section{Darstellung der Untersuchung}

In der Salus Klinik Lindow, einer stationären Entwöhnungseinrichtung für Alkoholabhängige im Land Brandenburg, wurden 103 alkoholabhängige Patienten in den ersten 14 Tagen während ihrer Behandlung mit verschiedenen Instrumenten zur Erfassung des Alkoholverlangens untersucht [20]. Die Diagnosestellung einer Alkoholabhängigkeit erfolgte über die Kriterien der ICD-10 und wurde durch die Ergebnisse des Composite International Diagnostic Interview (CIDI) bestätigt. Die Patienten waren im Mittel 39,7 Jahre alt ( $S D=7,26) .86 \%$ waren männlich. Von den Probanden lebten 47,6\% aktuell in einer Partnerschaft. Der Arbeitslosenanteil lag sehr hoch $(76,7 \%)$, die durchschnittliche Dauer der
Arbeitslosigkeit betrug 2,76 Jahre ( $S D=2,7)$. Weitere wichtige Stichprobenmerkmale sind in Tab. 4 zusammengestellt.

Die Untersuchung erstreckte sich über zwei Tage. Am ersten Tag nahmen die Patienten am Alkoholreagibilitätstest teil (Beschreibung des Ablaufs vgl. Abb.4), am nächsten Tag sollten die Patienten folgende Selbstbeurteilungsbogen ausfüllen: 1. LCS, 2. OCDS, 3. CBQ und 4. IDTSA.

\section{Ergebnisse}

\section{Ergebnisse im Alkoholreagibilitätstest}

Der Speichelfluss bei der Konfrontation mit Wasser (KW) betrug im Mittel 6,66 $\mathrm{g}(\mathrm{SD}=4,39)$, bei der Konfrontation mit Alkohol (KA) durchschnittlich 7,11 $\mathrm{g}(\mathrm{SD}=4,77)$. Der Unterschied von $0,45 \mathrm{~g}$ ist nicht signifikant $(\mathrm{p}=0,115)$. Deutliche Unterschiede lassen sich aber bei der körperlichen und kognitiven Reaktion bei der Wasser- bzw. Alkoholexposition finden. Es wurden deutlich ausgeprägtere körperliche Reak-

Tab. 3 Vergleich des Cravings vor und vier Monate nach einer stationären Entzugsbehandlung

\begin{tabular}{|c|c|c|c|c|c|}
\hline & \multicolumn{2}{|c|}{ abstinente Patienten $(\mathrm{n}=77)$} & \multicolumn{2}{|c|}{ trinkende Patienten $(n=84)$} & \multirow[t]{2}{*}{ Signifikanz (Manova) } \\
\hline & vor Entzug & $\begin{array}{l}4 \text { Monate } \\
\text { nach Entzug }\end{array}$ & vor Entzug & $\begin{array}{l}4 \text { Monate } \\
\text { nach Entzug }\end{array}$ & \\
\hline $\begin{array}{l}\text { gedrückte } \\
\text { Stimmungslage }\end{array}$ & $\begin{array}{l}M=12,6 \\
S D=8,9\end{array}$ & $\begin{array}{l}M=8,0 \\
S D=7,3\end{array}$ & $\begin{array}{l}M=16,4 \\
S D=10,1\end{array}$ & $\begin{array}{l}M=15,6 \\
S D=11,2\end{array}$ & $\begin{array}{l}\text { Trinkstatus } S S=2619,6 ; d f=1 ; F=26,6 ; \\
p=0,000 \\
\text { Veränderung } S S=587,7 ; d f=1 ; F=6,8 ; \\
p=0,010 \\
\text { Wechselwirkung } S S=295,8 ; d f=1 ; \\
F=0,3 ; p=0,066\end{array}$ \\
\hline $\begin{array}{l}\text { gehobene } \\
\text { Stimmungslage }\end{array}$ & $\begin{array}{l}M=7,3 \\
S D=4,3\end{array}$ & $\begin{array}{l}M=3,9 \\
S D=3,4\end{array}$ & $\begin{array}{l}M=8,3 \\
S D=4,8\end{array}$ & $\begin{array}{l}M=5,3 \\
S D=4,9\end{array}$ & $\begin{array}{l}\text { Trinkstatus } S S=116,6 ; d f=1 ; F=5,6 ; \\
p=0,019 \\
\text { Veränderung } S S=783,8 ; d f=1 ; \\
F=40,6 ; p=0,000 \\
\text { Wechselwirkung } S S=5,7 ; d f=1 ; F=0,3 ; \\
p=0,588\end{array}$ \\
\hline $\begin{array}{l}\text { Ärger und } \\
\text { Anspannung }\end{array}$ & $\begin{array}{l}M=5,8 \\
S D=4,6\end{array}$ & $\begin{array}{l}M=3,1 \\
S D=3,0\end{array}$ & $\begin{array}{l}M=7,4 \\
S D=5,3\end{array}$ & $\begin{array}{l}M=6,0 \\
S D=5,1\end{array}$ & $\begin{array}{l}\text { Trinkstatus } S S=409,5 ; d f=1 ; F=16,7 \\
p=0,000 \\
\text { Veränderung } S S=330,5 ; d f=1 \\
F=18,1 ; p=0,000 \\
\text { Wechselwirkung } S S=33,6 ; d f=1 \\
F=1,8 ; p=0,177\end{array}$ \\
\hline $\begin{array}{l}\text { Zufriedenheit } \\
\text { und Entspannung }\end{array}$ & $\begin{array}{l}M=3,5 \\
S D=2,8\end{array}$ & $\begin{array}{l}M=1,9 \\
S D=1,7\end{array}$ & $\begin{array}{l}M=3,9 \\
S D=2,7\end{array}$ & $\begin{array}{l}M=2,4 \\
S D=2,4\end{array}$ & $\begin{array}{l}\text { Trinkstatus } S S=116,58 ; d f=1 ; F=5,60 ; \\
p=0,019 \\
\text { Veränderung } S S=783,83 ; d f=1 ; \\
F=40,64 ; p=0,000 \\
\text { Wechselwirkung } S S=5,70 ; d f=1 ; \\
F=0,30 ; p=0,588\end{array}$ \\
\hline
\end{tabular}


In welchen Situationen und Stimmungen haben Sie Verlangen (Jieper, Gier) nach Alkohol in den letzten 30 Tagen verspürt? Bei jeder Aussage sollen Sie zunächst angeben, wie stark das Verlangen nach Alkohol war und dann ankreuzen, ob Sie in einer solchen Situation/Stimmung getrunken haben.

Unbekannt bedeutet, dass Sie eine solche Situation oder eine solche Stimmung noch nie erlebt haben.

Stimmungen/Situationen

1.Wenn ich in guter Stimmung war

2. Wenn ich mich angespannt fühlte

3. Wenn ich nicht schlafen konnte

4. Wenn ich mich allein und einsam fühlte

5. Wenn ich mich niedergeschlagen fühlte

6 . Wenn ich an schöne Rauscherlebnisse zurückdachte

7. Wenn ich abschalten wollte, um mich zu betäuben

8. Wenn ich mich geärgert hatte oder wütend war

9. Wenn ich es mir behaglich/ gemütlich machen wollte 10. Wenn ich Feierabend hatte

11. Wenn ich mich herabgesetzt behandelt fühlte

12. Wenn ich mir hilflos oder schwach vorkam

13. Wenn ich auf einer Feier war, auf der getrunken wurde

14. Wenn ich am Arbeitsplatz Ärger (z.B. mit Kollegen) hatte

15. Wenn ich mich in einer depressiven Stimmung fühlte

16. Wenn ich Angst verspürte 17. Wenn ich finanzielle Sorgen hatte

18. Wenn etwas erfolgreich verlaufen war

19. Wenn ich Probleme mit mir nahe stehenden Menschen hatte

20. Wenn ich unsicher war und mich nicht entscheiden konnte 21. Wenn ich mich körperlich sehr schlecht fühlte

22. Wenn ich zufrieden war, weil ich etwas erledigt hatte

23. Wenn ich beweisen wollte, dass ich trinken kann, ohne betrunken zu werden

24. Wenn ich unerwartet eine Flasche meines Lieblingsgetränkes fand

25 . Wenn ich meine sexuellen Gefühle steigern wollte 26. Wenn ich mich darüber ärgerte, dass etwas schief gelaufen war
Verlangen nach Alkohol

kannt nicht

Alkohol getrunken

sehr ja nein

stark

$\begin{array}{lllllll}1 & 2 & 3 & 4 & 5 & 2 & 1 \\ 1 & 2 & 3 & 4 & 5 & 2 & 1 \\ 1 & 2 & 3 & 4 & 5 & 2 & 1 \\ 1 & 2 & 3 & 4 & 5 & 2 & 1\end{array}$

1

1

1

1

1

1

1

$1-5$

$2-1-2$

2

3

4

1

$$
2
$$

3

4

5

1$$
1
$$

1

1

1

1

1

1

1$$
1
$$

2

3

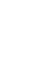

12

1
2

3

3

45

$\begin{array}{ll}2 & 1 \\ 2 & 1 \\ 2 & 1 \\ 2 & 1 \\ 2 & 1 \\ 2 & 1 \\ 2 & 1 \\ 2 & 1\end{array}$

Abb. 3 Auftreten von Craving (LCS) 
Tab. 4 Beschreibung der Untersuchungsstichprobe

\begin{tabular}{|c|c|c|}
\hline Variable & & $\begin{array}{l}\text { prozentualer } \\
\text { Anteil }(n=103)\end{array}$ \\
\hline Alter & $\begin{array}{l}\text { bis } 30 \text { Jahre } \\
31-40 \text { Jahre } \\
41-50 \text { Jahre } \\
51-65 \text { Jahre } \\
\text { über } 65 \text { Jahre }\end{array}$ & $\begin{array}{r}11,6 \% \\
42,8 \% \\
37,8 \% \\
6,0 \% \\
1,8 \%\end{array}$ \\
\hline Familienstand & $\begin{array}{l}\text { ledig } \\
\text { verheiratet } \\
\text { getrennt lebend } \\
\text { geschieden }\end{array}$ & $\begin{array}{l}31,1 \% \\
24,3 \% \\
12,6 \% \\
32,1 \%\end{array}$ \\
\hline Schulabschluss & $\begin{array}{l}\text { Sonderschule } \\
\text { Hauptschule } \\
\text { Realschule } \\
\text { Abitur }\end{array}$ & $\begin{array}{r}9,7 \% \\
18,4 \% \\
59,2 \% \\
12,6 \%\end{array}$ \\
\hline Wohnsituation & $\begin{array}{l}\text { ohne festen Wohnsitz } \\
\text { eigene Mietwohnung } \\
\text { eigenes Haus } \\
\text { bei Familienangehörigen, } \\
\text { im Heim }\end{array}$ & $\begin{array}{r}4,9 \% \\
61,2 \% \\
14,6 \% \\
19,4 \%\end{array}$ \\
\hline
\end{tabular}

tionen $(p=0,000)$ bei der Konfrontation mit Alkohol $(\mathrm{M}=1,82 ; \mathrm{SD}=2,58)$ gefunden als bei der Konfrontation mit Wasser $(M=1,03 ; S D=1,88)$. Gleiches gilt für die kognitiven Reaktionen, die nur bei $\mathrm{KA}(\mathrm{M}=2,03, \mathrm{SD}=2,74)$ und kaum bei der $\mathrm{KW}(\mathrm{M}=0,48 ; \mathrm{SD}=1,23)$ zu beobachten waren $(p=0,000)$. Es ließen sich keine signifikanten Korrelationen zwischen der Variablen „Speichelfluss“ mit kognitiven oder körperlichen Reaktionen finden.

\section{Ergebnisse im IDTSA und dem Alkoholreagibilitätstest}

Im IDTSA ergab sich weitgehend eine Übereinstimmung mit den statistischen Kennwerten der Stichprobe von Lindenmeyer und Florin [17]. Wie in Tab. 5 deutlich wird, findet sich keine signifikante Korrelation zwischen den Skalen des IDTSA und der Speichelflussdifferenz, wohl aber lassen sich signifikante Zusammenhänge mit den Unterschieden bezüglich körperlicher und kognitiver Reaktion feststellen. Es zeigt sich, dass Personen, die als spezifische Risikosituation „plötzliches Verlangen“ angeben, sowohl körperlich als auch kognitiv stärker bei der Konfrontation mit Alkohol reagieren.

Bei den intrapersonellen Risikosituationen besteht zusammenfassend ein signifikanter Zusammenhang mit körperlichen und kognitiven Reaktionen, bei interpersonellen Risikosituationen hingegen nicht.

\section{Ergebnisse im LCS und dem Alkoholreagibilitätstest}

Die durchschnittlichen Mittelwerte bei den vier Subskalen zum Craving sind in der Stichprobe der entwöhnungsbehandelten Alkoholabhängigen deutlich stärker ausgeprägt als in der Stichprobe von Veltrup [18]. Bei der Stärke des Verlangens in den letzten 30 Tagen vor dem Ausfüllen des Fragebogens hingegen gaben die entzugsbehandelten Patienten [18] mit $M=71,4$ deutlich höhere Werte an (möglicher Wertebereich 0-100) als die Untersuchungsstichprobe entwöhnungsbehandelter Patienten ( $M=63,38$; $S D=28,03$ ).

In Tab. 6 sind die Korrelationen zwischen den Variablen der LCS und den Variablen des Alkoholreagibilitätstests dargestellt. Bei der Häufigkeit des Verlangens liegt kein Intervallskalenniveau vor. Hier wurden die Korrelation mit Spearman's Rho berechnet.

Es zeigt sich erneut, dass keine signifikanten Zusammenhänge zwischen den abhängigen Variablen „Speichelfluss“ und „Veränderung körperlicher Reaktion“ und den Variablen des Lübecker Craving-Fragebogens zu finden sind. Für die abhängige Variable „Veränderung kognitiver Reaktion“ lässt sich ein hoch signifikanter Zusammenhang mit dem Faktor „Ärger/ Anspannung“ finden. Dieser Zusammenhang äußert sich in einer geringen positiven Korrelation. Das heißt, Personen, die in Situationen, in denen sie ärgerlich und angespannt sind, Verlangen haben, zeigen eine größere Veränderung in der kognitiven Reaktion beim Alkoholreagibilitätstest. Außerdem zeigt sich ein signifikanter Zusammenhang für die Variable „Stärke des Verlangens“ und die abhängige Variable „Veränderung kognitiver Reaktion“, der sich in einer geringen positiven Korrelation äußert. Das bedeutet, dass Personen, die für die letzten 30 Tage starkes Verlangen angaben, eine

\footnotetext{
1. Instruktions- und Übungsphase

Einführung in die Untersuchung, Förderung der Kooperationsbereitschaft, Beantwortung von Fragen der Probanden, Üben des Platzierens

von acht Wattestäbchen verschiedener Größe im Mund des Probanden

2. Konfrontation mit Wasser

Wiegen der acht Wattestäbchen, Platzieren der Wattestäbchen im Mund des Probanden, Präsentation eines Glases mit Wasser, Anleitung vom Tonband, Konfrontation von Wasser über 5 Minuten, Entfernen der Wattestäbchen, Wiegen der Wattestäbchen, Beantwortung eines Fragebogens ,Alkoholverlangen beim Anblick von Wasser“"

3. Konfrontation mit Alkohol“"

Wiegen der acht Wattestäbchen, Platzieren der Wattestäbchen im Mund des Probanden, Präsentation des alkoholischen Lieblingsgetränks des Probanden, Anleitung vom Tonband, Konfrontation mit Alkohol über 5 Minuten, Entfernen der Wattestäbchen, Beantwortung eines Fragebogens ,Alkoholverlangen beim Anblick von Alkohol“"

4 Auswertung

Als Ergebnis wird die Gewichtsdifferenz zwischen dem Speichelfluss beim Anblick von Alkohol und von Wasser festgehalten. Dazu werden die jeweiligen Gewichte vor und nach der Konfrontation mit der Substanz subtrahiert. Das Resultat ist die Menge an Speichel, die beim Anblick von Alkohol mehr bzw. weniger produziert wurde.
}

Abb. 4 Untersuchungsablauf beim Alkoholreagibilitätstest. 


\begin{tabular}{|c|c|c|c|}
\hline & $\begin{array}{l}\text { Differenz } \\
\text { Speichelfluss } \\
(\mathrm{n}=101)\end{array}$ & $\begin{array}{l}\text { Differenz } \\
\text { körperlicher Reaktion } \\
(n=100)\end{array}$ & $\begin{array}{l}\text { Differenz } \\
\text { kognitiver Reaktion } \\
(n=99)\end{array}$ \\
\hline angenehme Gefühle & 0,036 & 0,104 & 0,031 \\
\hline körperliche Beschwerden & 0,136 & $0,228 *$ & $0,227^{*}$ \\
\hline plötzliches Verlangen & 0,099 & $0,265 * *$ & $0,266 * *$ \\
\hline unangenehme Gefühle & 0,065 & $0,208 *$ & $0,243 *$ \\
\hline Geselligkeit & 0,114 & 0,024 & 0,152 \\
\hline soziale Konflikte & $-0,012$ & $0,201^{*}$ & $0,239 *$ \\
\hline soziale Verführung & 0,050 & 0,121 & 0,102 \\
\hline
\end{tabular}

Tab. 5 Korrelationskoeffizienten der Skalen des Inventory of Drug Taking Situations und den Variablen des Alkoholreagibilitätstests

* Die Korrelation ist auf dem Niveau von 0,05 (2-seitig) signifikant.

** Die Korrelation ist auf dem Niveau von 0,01 (2-seitig) signifikant.

\begin{tabular}{|c|c|c|c|}
\hline & $\begin{array}{l}\text { Differenz } \\
\text { Speichelfluss } \\
(n=98)\end{array}$ & $\begin{array}{l}\text { Differenz } \\
\text { körperliche Reaktion } \\
(n=97)\end{array}$ & $\begin{array}{l}\text { Differenz } \\
\text { kognitive Reaktion } \\
(n=96)\end{array}$ \\
\hline gedrückte Stimmung & 0,004 & 0,017 & 0,158 \\
\hline gehobene Stimmung & $-0,076$ & 0,000 & 0,157 \\
\hline Zufriedenheit/Entspannung & $-0,027$ & 0,002 & 0,033 \\
\hline $\begin{array}{l}\text { Stärke des Verlangens } \\
\text { (letzten } 30 \text { Tage) }\end{array}$ & $-0,055$ & 0,158 & $0,229^{*}$ \\
\hline $\begin{array}{l}\text { Häufigkeit des Verlangens } \\
\text { (letzten } 30 \text { Tage) }\end{array}$ & 0,085 & $-0,019$ & 0,146 \\
\hline
\end{tabular}

Tab. 6 Korrelationskoeffizienten der Variablen der Lübecker Craving Skala und des Alkoholreagibilitätstests

\footnotetext{
* Die Korrelation ist auf dem Niveau von 0,05 (2-seitig) signifikant.

** Die Korrelation ist auf dem Niveau von 0,01 (2-seitig) signifikant.
}

höhere Veränderung in der kognitiven Reaktion bei der Konfrontation mit Alkohol aufweisen.

\section{Ergebnisse in der OCDS und dem Alkoholreagibilitätstest}

Für den Gesamtwert der Obsessive Compulsive Drinking Scale wurden Mittelwert und Standardabweichung berechnet. Außerdem wurde mittels Kolmogorov-Smirnov-Test geprüft, ob die Daten normalverteilt sind. Es zeigt sich, dass der hier gewonnene Mittelwert $(M=16,87 ; S D=8,10)$ etwas unter dem Mittelwert $(M=22,5)$ der Untersuchung von Anton, Moak und Latham [6] liegt.

Es ist erkennbar, dass sich keine Zusammenhänge zwischen den abhängigen Variablen „Speichelfluss“ und „Veränderung körperlicher Reaktion“ mit der Zwanghaftigkeit der Obsessive Compulsive Drinking Scale finden lassen. Ein hoch signifikantes Ergebnis zeigt sich bei der Korrelation der Zwanghaftigkeit mit der kognitiven Reaktion. Hier liegt ein gering positives Ergebnis vor, das besagt, dass Personen mit einer hohen Zwanghaftigkeit des Verlangens bei der Konfrontation mit Alkohol kognitiv stärker reagieren.

\section{Ergebnisse im CBQ und dem Alkoholreagibilitätstest}

Da sich zum Untersuchungszeitpunkt keine Hinweise zur Auswertung, zu Gütekriterien und zur Struktur des Fragebogens finden ließen, wurde zuerst eine ausführliche Analyse des Fragebogens durchgeführt. Begonnen wurde mit der
Berechnung von Mittelwerten, Standardabweichungen und Schwierigkeitsindizes für die Einzelitems. Letztere lagen alle im Bereich von 20 bis 80, es wurden für die weitere Analyse keine Items eliminiert. Anschließend erfolgte die Berechnung der Normalverteilung mittels Kolmogorov-Smirrnov-Test. Diese ergab, dass die Daten aller Einzelitems nicht normalverteilt sind. Im Folgenden wurde zur Klärung der internen Struktur des Fragebogens eine Faktorenanalyse berechnet. Als Extraktionsmethode wurde die Hauptkomponentenmethode verwendet, als Rotationsmethode die Varimax-Methode (eine orthogonale Rotationstechnik) mit Kaiser-Normalisierung. Da zunächst keine Hinweise auf die Anzahl möglicher Faktoren vorlagen, wurde keine Eingrenzung der Faktorenzahl vorgenommen. Es ergab sich, dass vier Faktoren einen Eigenwert über 1 erreichten. Bei einer 4-Faktoren-Lösung ließen sich $69,6 \%$ der Varianz der 20 Variablen erklären, bei einer

Tab. 7 Korrelationskoeffizienten der Zwanghaftigkeit der Obsessive Compulsive Drinking Scale und den Variablen des Alkoholreagibilitätstests

\begin{tabular}{llll}
\hline & $\begin{array}{l}\text { Differenz } \\
\text { Speichelfluss }\end{array}$ & $\begin{array}{l}\text { Differenz } \\
\text { körperlicher } \\
\text { Reaktion } \\
(\mathrm{n}=95)\end{array}$ & $\begin{array}{l}\text { Differenz } \\
\text { kognitiver } \\
\text { Reaktion } \\
(\mathrm{n}=93)\end{array}$ \\
\hline Zwanghaftigkeit & 0,114 & 0,145 & $0,298^{*}$
\end{tabular}

* Die Korrelation ist auf dem Niveau von 0,01 (2-seitig) signifikant. 
3-Faktoren-Lösung 64,2\% und bei einer 2-Faktoren-Lösung 57,5\%. Es zeigt sich, dass anhand dieser Ergebnisse keine eindeutige Auswahl einer Faktorenlösung erfolgen kann. Daher wurden für alle drei Faktorenlösungen die Faktorenladungen der rotierten Faktormatrix berechnet. Anhand dieser Matrizen konnte eine inhaltliche Bewertung der extrahierten Faktoren durchgeführt werden. Dabei zeigt sich, dass die 3-Faktoren-Lösung am besten zu interpretieren ist. Allerdings mussten fünf Items $(1,6,17,20,11)$ eliminiert werden, da sie auf zwei Faktoren annähernd gleich hoch luden. Die restlichen Items lassen sich zu drei Faktoren zusammenfassen: „Übermächtigkeit und Zwanghaftigkeit des Verlangens“, „Suchtmittelkonsum als Bewältigung von Verlangen“ sowie
„Dauerhaftigkeit des Verlangens“. Anschließend erfolgte die Bestimmung der internen Konsistenz der Faktoren (mittels Cronbach's Alpha) und die Berechnung der Trennschärfekoeffizienten für die Einzelitems (siehe Tab.8). Bei der Berechnung der Trennschärfekoeffizienten zeigten sich gute Ergebnisse, es musste kein Item aufgrund seiner $\mathrm{zu}$ geringen Trennschärfe eliminiert werden. Die Bestimmung von Cronbach's Alpha ohne das jeweilige Einzelitem ergab nur beim Item 10 („Das Verlangen ist meine Strafe dafür, dass ich süchtig bin“) eine Erhöhung der internen Konsistenz der Subskala „Suchtmittel als Bewältigung von Verlangen“. Daher wurde beschlossen, dieses Item zu eliminieren. Anschließend wurde für alle drei Subskalen Cronbachs Alpha berechnet

Tab. 8 Faktorenstruktur des Cravings Beliefs Questionnaire

\begin{tabular}{|c|c|c|c|c|}
\hline Item-Nr. & Item & Faktorladung & Schwierigkeit & Trennschärfe \\
\hline 3 & Das Verlangen kann einen verrückt machen. & 0,80 & 0,62 & 0,70 \\
\hline 2 & Wenn ich das Verlangen nicht stoppe, wird es immer schlimmer. & 0,77 & 0,75 & 0,55 \\
\hline 4 & Ich nehme nur Suchtmittel, weil das Verlangen so stark ist. & 0,74 & 0,55 & 0,73 \\
\hline 7 & $\begin{array}{l}\text { Wenn das Verlangen einmal angefangen hat, habe ich keine Kontrolle } \\
\text { mehr darüber. }\end{array}$ & 0,71 & 0,56 & 0,78 \\
\hline 13 & $\begin{array}{l}\text { Das Verlangen macht mich so nervös, dass ich es einfach nicht mehr } \\
\text { aushalten kann. }\end{array}$ & 0,66 & 0,49 & 0,75 \\
\hline 16 & $\begin{array}{l}\text { Wenn ich wirklich ganz starkes Verlangen verspüre, dann bin ich zu gar } \\
\text { nichts mehr fähig. }\end{array}$ & 0,60 & 0,43 & 0,70 \\
\hline 12 & $\begin{array}{l}\text { Die Vorstellungen und Gedanken, die ich habe, wenn das Verlangen } \\
\text { nach Suchtmitteln stark ist, unterliegen nicht meiner Kontrolle. }\end{array}$ & 0,57 & 0,48 & 0,71 \\
\hline 9 & $\begin{array}{l}\text { Ich kann die körperlichen Symptome, die mit dem Verlangen } \\
\text { einhergehen, nicht aushalten. }\end{array}$ & 0,56 & 0,44 & 0,72 \\
\hline
\end{tabular}

Faktor 2: Suchtmittelkonsum als Bewältigung von Verlangen $\mathrm{n}=$ 99; $\mathrm{M}=6,78 ; \mathrm{SD}=4,19 ;$ Alpha $=0,78=$ > keine Normalverteilung $(\mathrm{KSZ}=1,55 ; \mathrm{p}=0,016)$

Item-Nr. Item

19

18

15
Wenn man starkes Verlangen verspürt, dann ist es in Ordnung, wenn man Suchtmittel nimmt.
Faktorladung Schwierigkeit

0,78

Wenn das Verlangen zu stark wird, sind Suchtmittel die einzige Lösung, 0,77 um mit dem Problem fertig zu werden.

Ich nehme nur Suchtmittel, weil das Verlangen so stark ist.
0,61
0,32

0,46

0,26
Trennschärfe

0,66

0,69

0,56

Trennschärfe

0,76

0,73

0,71

\begin{tabular}{llll}
\hline & $\begin{array}{l}\text { Differenz } \\
\text { Speichelfluss } \\
(\mathrm{n}=99)\end{array}$ & $\begin{array}{l}\text { Differenz } \\
\text { körperlicher Reaktion } \\
(\mathrm{n}=98)\end{array}$ & $\begin{array}{l}\text { Differenz } \\
\text { kognitiver Reaktion } \\
(\mathrm{n}=97)\end{array}$ \\
\hline Übermächtigkeit und Zwanghaftigkeit des Verlangens & 0,170 & 0,159 & $0,273^{* *}$ \\
$\begin{array}{l}\text { Suchtmittel als Bewältigung von Verlangen } \\
\text { Dauerhaftigkeit des Verlangens }\end{array}$ & $\begin{array}{l}0,236^{*} \\
0,142\end{array}$ & $-0,189$ & 0,113 \\
\hline
\end{tabular}

Tab. 9 Korrelationskoeffizienten der Subskalen des Craving Beliefs Questionnaire und den Variablen des Alkoholreagibilitätstests

\footnotetext{
* Die Korrelation ist auf dem Niveau von 0,01 (2-seitig) signifikant.

** Die Korrelation ist auf dem Niveau von 0,05 (2-seitig) signifikant.
} 
(siehe Tab. 8). Dabei zeigt sich, dass die Faktoren intern konsistent sind ( $\mathrm{a}=0,78$ bis 0,91 ).

Die Bestimmung der Interkorrelationen der Subskalen ergab signifikante Zusammenhänge mittlerer Stärke (zwischen 0,51 und 0,58) zwischen allen drei Faktoren. Des Weiteren wurden nun Mittelwerte und Standardabweichungen für die einzelnen Faktoren berechnet. Außerdem wurden die Daten der Subskalen mittels Kolmogorov-Smirrnov-Test auf Normalverteilung geprüft. Hier zeigt sich, dass die Daten des Faktors „Übermächtigkeit und Zwanghaftigkeit des Verangens“ normalverteilt sind, für die Daten der anderen beiden Subskalen ließ sich keine Normalverteilung feststellen. Die Ergebnisse zu den Berechnungen bezüglich der Zusammenhänge zwischen den Subskalen des Craving Beliefs Questionnaire und den Variablen des Alkoholreagibilitätstests mittels der Pearson-Korrelation finden sich in Tab. 9.

Es lassen sich keine signifikanten Korrelationen für die Variablen des Craving Beliefs Questionnaire und der abhängigen Variablen „Veränderung körperlicher Reaktion“ finden. Für die abhängige Variable „Speichelfluss“ zeigt sich ein signifikanter, gering positiver Zusammenhang mit der Variable „Suchtmittel als Bewältigung von Verlangen“. Das heißt, dass Personen, für die es in Ordnung ist, aufgrund von Verlangen Suchtmittel zu nehmen, eine stärkere Veränderung im Speichelfluss bei der Konfrontation mit Alkohol aufweisen. Eine hoch signifikante Korrelation lässt sich für die Variable „Veränderung kognitiver Reaktion“ und den Faktor „Übermächtigkeit und Zwanghaftigkeit des Verlangens“ zeigen. Dieser gering positive Zusammenhang bedeutet, dass Personen mit vielen bzw. starken Grundüberzeugungen zur Übermächtigkeit und Zwanghaftigkeit ihres Verlangens bei der Konfrontation mit Alkohol kognitiv stärker reagieren.

\section{Diskussion}

Craving ist ein vielschichtiges und komplexes Konstrukt, die theoretische Fundierung und empirische Erfassung bleibt schwierig. Die vorgestellte Studie hat versucht, verschiedene Instrumente bei der Erhebung des Cravings zu nutzen. Die Resultate im Alkoholreagibilitätstest zeigen, dass sich bei der Messung des Speichelflusses bei alkoholabhängigen Probanden keine Unterschiede unter den verschiedenen Versuchsbedingungen ergeben. Möglicherweise ist dieses Ergebnis durch den Testaufbau beeinflusst. So ist plausibel, dass der Speichelfluss bei der Alkoholkonfrontation durch die vorherige Messung reduziert ist, da der Mund bei der ersten Übung mit Wasser wohl nicht so trocken war wie nach diesem Versuchsteil. Bei den körperlichen und kognitiven Reaktionen zeigen sich Unterschiede. Es bleibt zu klären, ob es sich bei den verstärkten körperlichen Reaktionen bei der Alkoholexposition um ein verlangensspezifisches Phänomen handelt, oder um eine allgemeine Erhöhung des Erregungsniveaus durch die Nähe des alkoholischen „Lieblingsgetränkes“.

Interessant sind die gefundenen Zusammenhänge zwischen dem Ausmaß von kognitiver und körperlicher Reaktion beim Alkoholreagibilitätstest und den Ergebnissen in den Selbstbeurteilungsskalen zum Craving. Personen, die vermehrt intrapersonelle Risikosituationen im IDTSA beschreiben, weisen auch stärkere kognitive und körperliche Reaktionen auf. Auch die subjektiv erlebte Stärke des Alkoholverlangens in den letzten 30 Tagen vor dem Alkoholreagibilitätstests korreliert mit dem Ausmaß an kognitiven Reaktionen bei dem konkreten Versuch.

Weiter steht die Zwanghaftigkeit des Alkoholverlangens in einem Zusammenhang mit dem Craving bei der Konfrontation mit Alkohol. Je stärker die Grundüberzeugungen bezüglich der Macht (Übermächtigkeit und Zwanghaftigkeit) von Alkoholverlangen sind, desto ausgeprägter ist die kognitive Reaktion bei der Konfrontation mit Alkohol. Interessant ist, dass die Grundüberzeugung, wonach die Einnahme von Suchtmitteln in Ordnung ist, wenn Verlangen auftritt, mit dem vermehrten Speichelfluss zusammenhängt. Solche Probanden könnten laut dem Modell von Beck et al. [5] kurz davor sein, ihr Verhalten umzusetzen. Die Erfassung der vielfältigen Bedingungen zum Craving sind sehr wohl auch bedeutsam für die Therapie. Insbesondere wurden von einer Reihe von Autoren Expositionsverfahren in vivo in der Behandlung von Suchtmittelabhängigen eingesetzt [21,22]. Die Übungssituationen werden durch die Konfrontation mit persönlich relevanten Auslösebedingungen (z.B. die Konfrontation mit alkoholischen Getränken, das Aufsuchen von früheren Trinkorten) so gestaltet, dass eine erhebliche Versuchung oder Verführung, Alkohol einzunehmen, entsteht [23]. In der Untersuchung von Veltrup [24] konnte gezeigt werden, dass bei einem großen Teil der wieder trinkenden Patienten in einer solchen Situation subjektiv ein Alkoholcraving wahrgenommen wird. Ziel der Expositionsübungen ist, dass Abstinente selbst bei starkem Verlangen nach Suchtmitteln in einer persönlichen Risikosituation standhalten können und dadurch Zugang zu neuen Bewältigungsmöglichkeiten und Selbstvertrauen finden. [25]. Dies gilt v.a. für das Bewältigen von intrapersonellen Risikosituationen. Erste Studien sprechen für die Wirksamkeit von Exposition in vivo bei der Behandlung von Suchtmittelabhängigen [26].

\section{Literatur}

${ }^{1}$ Watzl H, Gutbrod K. Verlangen nach Alkohol - Begriffsbestimmung, empirische Befunde und Erklärungsansätze. Suchtgefahren $1983 ; 29$ : 19-27

${ }^{2}$ Stockwell T. Is there a better word than „Craving“? British Journal of Addiction 1987; 82: 45-46

${ }^{3}$ Tiffany ST. A cognitive model of drug urges and drug-use behavior: role of automatic and nonautomatic processes. Psychological Review 1990; 97: 147-168

${ }^{4}$ Marlatt GA. Craving notes. British Journal of Addiction 1987; 82: 42-44

${ }^{5}$ Beck AT, Wright D, Newman C, Liese B. Kognitive Therapie der Sucht. Weinheim: Beltz, 1997

${ }^{6}$ Anton RF, Moak DH, Latham PK. The Obsessive Compulsive Drinking Scale. A self-rated instrument for the quantification of thoughts about alcohol and drinking behaviour. Alcoholism: Clinical and Experimental Research 1995; 19: 92-100

${ }^{7}$ Internationale Klassifikation psychischer Störungen. ICD-10 Kapitel V (F). In: Dilling H, Mombour W, Schmidt MH (Hrsg). Klinisch-diagnostische Leitlinien. Bern: Huber, 1991

${ }^{8}$ Wetterling T, Veltrup C, Junghanns K. Empirische Befunde zum umstrittenen Konzept Craving. In: Beutel M (Hrsg). Medikamentöse Behandlung der Alkoholkrankheit. Bundesverband für stationäre Suchtkrankenhilfe.. Geesthacht: Neuland, 1998: $72-87$ 
${ }^{9}$ Podschus J, Kuhn S, Gräf KJ, Rommelspacher H, Schmidt LG. Alkoholverlangen und dopaminerge Sensitivität: Untersuchungen mit Apomorphin. Sucht 1992; 38: 97-99

${ }^{10}$ Wetterling T, Veltrup C, Junghanns K. How to asses craving for alcohol. European Addiction Research 1997; 3: 110-115

${ }^{11}$ Wetterling T, Veltrup C, Junghanns K. Craving - ein ausreichend fundiertes Konzept? Fortschritte Neurologie Psychiatrie 1996; 64: $142-152$

${ }^{12}$ Ludwig AM, Wikler A, Stark LH. The first drink. Psychobiological aspects of craving. Archives of General Psychiatry 1974; 30 : 39-547

${ }^{13}$ Baker LH, Cooney NL, Pomerleau . Craving for alcohol: theoretical processes and treatment procedures. In: Cox WM (Hrsg). Treatment and Prevention of Alcohol Problems: a Resource Manual, 1987: 183-201

${ }^{14}$ Hughes RJ. Craving a psychological Construct. British Journal of Addication 1987; 16: 38-39

${ }^{15}$ Anton RF, Moak DH, Latham PK. The Obsessive Compulsive Drinking Scale. A new method of assessing outcome in alcoholism treatment studies. Archives of General Psychiatry 1996; 53 : 225-231

${ }^{16}$ Annis HN, Martin G, Graham JM. Inventory of Drug Taking Situations (Alcohol) IDTSA. User's guide. Toronto: Addiction Research Foundation, 1992

${ }^{17}$ Lindenmeyer J, Florin I. Testgütekriterien einer deutschen Version des Inventory of Drug Taking Situations für Alkoholabhängige (IDTSA). Verhaltenstherapie 1998; 8: 26-36

${ }^{18}$ Veltrup C. Erfassung des Craving bei Alkoholabhängigen mit Hilfe eines neuen Fragebogens (Lübecker Craving-Risiko-Rückfall-Fragebogen). Wiener klinische Wochenschrift 1994; 106: 75-79

${ }^{19}$ Veltrup C, Wetterling T. Die psychometrische Erfassung des Craving bei entzugsbehandelten Alkoholabhängigen. In: Mann K, Buchkremer G (Hrsg). Sucht - Grundlagen, Diagnostik, Therapie. Stuttgart: Fischer, 1996: 195-202

${ }^{20}$ Einsle F. Bedingungen für Alkoholverlangen. Diplom-Arbeit am Fachbereich Psychologie der Technischen Universität Dresden (unveröffentlicht), 1999

${ }^{21}$ Brandon TH, Piasecki TN, Quinn EP, Baker TB. Cue exposure treatment in nicotine dependence. In: Drummond DC, Tiffany ST, Glautier S, Remington B (Hrsg). Addictive behaviour. Cue exposure theory and practice. Chichester: Wiley, 1995: 211-228

22 Dave S, Powell JH. Cue exposure treatment in opiate and cocaine dependence. In: Drummond DC, Tiffany ST, Glautier S, Remington B (Hrsg). Addictive behaviour. Cue exposure theory and practice. Chichester: Wiley, 1995: 197-210

${ }^{23}$ Lindenmeyer J. Führe Dich in Versuchung ... Neue Wege der Rückfallbehandlung. Suchtreport 1995; 1: 38-43

${ }^{24}$ Veltrup C. Abstinenzgefährdung und Abstinenzbeendigung von Alkoholabhängigen nach einer umfassenden stationären Entzugsbehandlung Münster: Waxmann, 1995

${ }^{25}$ Drummond DC, Tiffany SG, Glautier S, Remington B. Cue exposure in understanding and treating addictive behaviours. In: Drummond DC, Tiffany ST, Glautier S (Hrsg). Addictive behaviour: Cue exposure theory and practice. Chichester: Wiley, 1995: $1-20$

${ }^{26}$ Rohsenow DJ, Monti PM, Abrams DB. Cue exposure treatment in alcohol dependence. In: Drummond DC, Tiffany ST, Glautier S, Remington B (Hrsg). Addictive behaviour: Cue exposure theory and practice. Chichester: Wiley, 1995: 168-196
XDr. phil. Clemens Veltrup

Therapieverbund Ostsee

Weidenweg 9-15

23562 Lübeck

E-mail: veltrup@tvo.kte-ag.de 